\title{
"Next Is Nexus" - A Retrospective Study To Check The Usefulness Of The Nexus Criteria Decision Instrument For Clearing The Cervical Spine Injury Following Blunt Trauma.
}

\author{
Dr Arnab Sinha ${ }^{1}$,Dr Santosh Kumar ${ }^{2}$, Dr Riteshrunu ${ }^{3}$ \\ Senior Resident, 2. Additional Professor, 3. Associate Professor, Department Of Orthopaedics, Indira Gandhi \\ Institute Of Medical Sciences, Patna,Bihar.
}

\begin{abstract}
Fear of failure to identify cervical spine injury has led to extremely liberal use of radiography in patients with blunt trauma and remotely possible neck injury. Eliminating even a small proportion of the cervical-spine radiographs ordered for patients with blunt trauma could lead to substantial savings and decrease patients' exposure to ionizing radiation. The National Emergency X-Radiography Utilization Study (NEXUS) is a very large, federally supported, multicenter, prospective study designed to define the sensitivity, for detecting significant cervical spine injury, of criteria previously shown to have high negative predictive value. We did a retrospective study of 100 case records to find out the usefulness of the NEXUS criteria decision instrument for clearing the cervical spine injury following blunt trauma.The sensitivity and specificity was 95,17,92 and 15 in the all patients group and the patients with clinically significant injury group, respectively. We recommend the routine use of the NEXUS criteria decision instrument in blunt injuries to clear the cervical spine in the Emergency Department.
\end{abstract}

Keywords: NEXUS, Cspine clearance, blunt Trauma

\section{Introduction}

Fear of missing a clinically occult injury has prompted physicians to order images of the cervical spine for virtually all patients who have blunt trauma. As a result, for each injury detected, a large number of films with negative findings are ordered. Because of the consequent human and economic losses, combined with medicolegal issues and concern about quality assurance, validation of selective criteria for ruling out probable cervical-spine injury requiring radiography in patients with blunt trauma is an important priority. The National Emergency X-Radiography Utilization Study (NEXUS) is a very large, federally supported, multicenter, prospective study designed to define the sensitivity, for detecting significant cervical spine injury, of criteria previously shown to have high negative predictive value.

AIM-To check the usefulness of the NEXUS criteria decision instrument for clearing the cervical spine injury following blunt trauma.

\section{Material And Methods}

E did a retrospective study of the case records of last 100 patients who had presented at the trauma centre of our hospital following a blunt trauma and who had been advised a cervical spine Xray independently without putting in use the NEXUS criteria decision instrument and in whom the criteria was put to use independent of being advised the Xray or not.The presence or absence of the criteria and the presence or absence of CSI on the final imaging findings were analysed. Thedemographic data for each study patient noted. For criteria that of -" could not be assessed" considered not to have met that criterion. The results of all the evaluations had been recorded on data forms before imaging of the cervical spine. Every patient with a completed data form underwent imaging.

Inclusion Criteria-All the patients with blunt trauma who underwent radiography of the cervical spine were included in the study.

1. penetrating trauma,

\section{Exclusion criteria}

2. Age $<18$ years, 3.those who underwent cervical-spine imaging for any other reason, unrelated to trauma, 4 . Patients Unwilling to participate in the study.

C spine Radiography-A standard series of three views of the cervical spine (cross-table lateral view, anteroposterior view, and open-mouth view of the odontoid) was obtained in all patients.

The NEXUS criteria decision instrument-

1.the absence of tenderness at the posterior midline of the cervical spine,

2. the absence of a focal neurologic deficit, 
"Next is NEXUS" - A retrospective study to check the usefulness of the....

3.a normal level of alertness, 4. no evidence of intoxication, and

5. absence of clinically apparent pain that might distract the patient from the pain of a cervical-spine injury. Patients who met all five criteria are considered to have a low probability of injury and not to require radiographic or other imaging.

Imaging assessment-Injuries that were not clinically significant were those that typically require no specific treatment and those that, if not identified, would be expected to result in no harm. Radiographically documented cervical-spine injuries were categorized as not clinically significant only if they were isolated and there was no evidence of other bony injury or ligamentous or spinal cord injury. All other cervical-spine injuries were considered clinically significant.

Study Analysis-A true positive result - patients who did not meet all five of the criteria and who were reported to have a radiographically documented cervical-spine injury. A false negative- patients who did not meet one or more of the five criteria and who had a radiographically documented injury. The result was true negative for patients who met all the criteria for a low probability of injury and who had no evidence of cervical-spine injury on radiography, whereas it was false positive for those who did not meet all the criteria but who had no injury.

\section{Results}

The mean age of the patients was 40 years (range 22 to 72 years), predominantly male (62 patients), maximum of them presenting to us after 8 hours of injury (84). The sensitivity and specificity was 95,17,92 and 15 in the all patients group and the patients with clinically significant injury group, respectively.

\begin{tabular}{|l|l|}
\hline All patients & \\
\hline SENSITIVITY & 95 \\
\hline SPECIFICITY & 17 \\
\hline Patients with clinically significant injury & \\
\hline SENSITIVITY & 92 \\
\hline SPECIFICITY & 15 \\
\hline
\end{tabular}

\section{Conclusion}

Eliminating even a small proportion of the cervical-spine radiographs ordered for patients with blunt trauma could lead to substantial savings and decrease patients' exposure to ionizing radiation.Clinicians can generally adhere to the clinical criteria in the decision instrument, but they should be free to make exceptions for individual patients on clinical grounds. In any case, no decision instrument is ever likely to be 100 percent sensitive, and the medical and economic costs of a quixotic search for absolute diagnostic certainty can lead to more harm than good.The sensitivity of this set of criteria approaches 100 percent for clinically important injuries, and its general application should result in both clinical and economic benefit. As with any other clinical tool, it should be applied with great care and should not replace clinical judgment in the care of individual patients. There may be compelling reasons to order cervical-spine images in individual cases, even if all the criteria for a low probability of injury are met. This criteria could lead to substantial health care savings as well as reduced periods of immobilization in crowded emergency departments hence, we recommend the routine use of the Nexus criteria decision instrument in blunt injuries to clear the cervical spine in the Emergency Department.

\section{Discussion/ Review Of Literature}

Fear of failure to identify cervical spine injury has led to extremely liberal use of radiography in patients with blunt trauma and remotely possible neck injury. There is considerable variation in published guidelines and also among physicians with respect to the use of radiography.Cervical-spine radiography is a "little-ticket" item - a low-cost procedure that adds substantially to health care costs because of the high volume of its use.Furthermore, patients are often immobilized on a backboard for many hours while awaiting radiography, leading to considerable discomfort and unnecessary use of space in crowded emergency departmentsDue to the unclarity of whether each of the 5 individual criteria is necessary for the decision instrument to maintain its high sensitivity, a substudy to examine the NEXUS database to determine the contribution of each of the 5 individual low-risk clinical criteria to the overall sensitivity of the decision instrument was done. The result showed that all but 8 of 818 patients with CSI, and all but 2 of 578 patients with significant CSI, were identified by using the decision instrument. A substantial number of patients with CSI (236/818 [29\%]) and patients with significant CSI (175/578 [30\%]) met only 1 of the 5 non-low-risk criteria, and each of the 5 criteria was the only indicator of non-low-risk status in at least 8 patients with CSI and at least 5 patients with significant CSI. It concluded that because each of the 5 low-risk criteria was the only marker of non-low-risk status in at least a few patients with significant CSI, modification of the overall NEXUS decision 
instrument by eliminating any one of the criteria would markedly reduce sensitivity and make the instrument unacceptable for clinical use.

Since studies of cervical spine injury involve individual institutions or special populations and there is little reliable information regarding natural cervical spine injury patterns after blunt trauma. A substudy of the National Emergency X-Radiography Utilization Study project was designed to accurately assess the prevalence, spectrum, and distribution of cervical spine injury after blunt trauma. It prospectively enrolled all patients with blunt trauma undergoing cervical spine radiography at 21 diverse institutions. Injury status was determined by review of all radiographic studies obtained on each patient. For each individual injury, which specific films revealed the injury, the level and location of injury on each vertebra, and the age and sex of the patient was recorded.The results showed of the 34,069 enrolled patients with blunt trauma, $818(2.4 \%)$ individuals had a total of 1,496 distinct cervical spine injuries to 1,285 different cervical spine structures. The second cervical vertebra was the most common level of injury (286 [24.0\%] fractures, including 92 odontoid fractures), and 470 (39.3\%) fractures occurred in the 2 lowest cervical vertebrae (C6 and C7). The vertebral body, injured in 235 patients, was the most frequent site of fracture and nearly one third of all injuries $(29.3 \%)$ were considered clinically insignificant. It was concluded that Cervical spine injuries occur in a small minority of patients with blunt trauma who undergo imaging. The atlantoaxial region is the most common site of injury, and the sixth and seventh vertebrae are involved in over one third of all injuries. Other spine levels are much more commonly involved than has previously been appreciated. A substantial minority of radiographically defined cervical spine injuries are of little clinical importance.Anotherprospective cohort study done to evaluate the accuracy, reliability, and potential impact of the National Emergency X-Radiography Utilization Study (NEXUS) low-risk criteria for cervical spine radiography, when applied in 10 Canadian emergency departments (EDs),that recruited 8,924 alert and stable adult trauma patients it was found out that 151 (1.7\%) patients had an important cervical spine injury. The combined NEXUS criteria identified important cervical spine injury with a sensitivity of $92.7 \%$ (95\% CI $87 \%$ to $96 \%$ ) and a specificity of $37.8 \%$ (95\% CI 37\% to $39 \%)$. Application of the NEXUS criteria would have potentially reduced cervical spine radiography rates by $6.1 \%$ from the actual rate of $68.9 \%$ to $62.8 \%$. This group concluded that the NEXUS low-risk criteria to be less sensitive than previously reported and that the NEXUS low-risk criteria should be further explicitly and prospectively evaluated for accuracy and reliability before widespread clinical use outside of the United States.

Pediatric victims of blunt trauma have developmental and anatomic characteristics that can make it difficult to assess their risk of cervical spine injury (CSI). Retrospective report have not identified any cases of CSI in either children or adults in the absence of neck pain, neurologic symptoms, distracting injury, or altered mental status. Hence, a study was done to examine the incidence and spectrum of spine injury in patients who are younger than 18 years and to evaluate the efficacy of the National Emergency X-Radiography Utilization Study (NEXUS) decision instrument for obtaining cervical spine radiography in pediatric trauma victims.A prospective, multicenter study to evaluate pediatric blunt trauma victims was done. All patients who presented to participating emergency departments underwent clinical evaluation before radiographic imaging. The presence or absence of the following criteria was noted: midline cervical tenderness, altered level of alertness, evidence of intoxication, neurologic abnormality, and presence of painful distracting injury. Presence or absence of each individual criterion was documented for each patient before radiographic imaging, unless the patient was judged to be too unstable to complete the clinical evaluation before radiographs. The decision to radiograph a patient was entirely at the physician's discretion and not driven by the NEXUS questionnaire. The presence or absence of CSI was based on the final interpretation of all radiographic studies. Data on all patients who were younger than 18 years were sequestered from the main database for separate analysis.

The results showed that there were 3065 patients (9.0\% of all NEXUS patients) who were younger than 18 years in this cohort, 30 of whom $(0.98 \%)$ sustained a CSI. Included in the study were 88 children who were younger than 2, 817 who were between 2 and 8 , and 2160 who were 8 to 17 . Fractures of the lower cervical vertebrae (C5-C7) accounted for $45.9 \%$ of pediatric CSIs. No case of spinal cord injury without radiographic abnormality was reported in any child in this study, although 22 cases were reported in adults. Only 4 of the 30 injured children were younger than 9 years, and none was younger than 2 years. Tenderness and distracting injury were the 2 most common abnormalities noted in patients with and without CSI. The decision rule correctly identified all pediatric CSI victims (sensitivity: $100.0 \%$; 95\% confidence interval: $87.8 \%-100.0 \%$ ) and correctly designated 603 patients as low risk for CSI (negative predictive value: 100.0\%; 95\% confidence interval: $99.4 \%-100.0 \%$ ).It concluded that the lower cervical spine is the most common site of CSI in children, and fractures are the most common type of injury. CSI is rare among patients aged 8 years or younger. The NEXUS decision instrument performed well in children, and its use could reduce pediatric cervical spine imaging by nearly $20 \%$. However, the small number of infants and toddlers in the study suggests caution in applying the NEXUS criteria to this particular age group.A study was done to better define the incidence and characteristics of patients with spinal cord injury without radiographic abnormality (SCIWORA), using the database of the National Emergency X-Radiography Utilization Study (NEXUS).It was a prospective, 
observational study of blunt trauma patients in 21 U.S. medical centers undergoing plain cervical radiography. SCIWORA was defined as spinal cord injury demonstrated by magnetic resonance imaging, when a complete, technically adequate plain radiographic series revealed no injury.The results showed that of the 34,069 patients entered, there were 818 (2.4\%) with cervical spine injury, including $27(0.08 \%)$ patients with SCIWORA. Over 3,000 children were enrolled, including 30 with cervical spine injury, but none had SCIWORA. The most common magnetic resonance imaging findings among SCIWORA patients were central disc herniation, spinal stenosis, and cord edema or contusion. Central cord syndrome was described in 10 cases. This study concluded that in the large NEXUS cohort, SCIWORA was an uncommon disorder, and occurred only in adults.

\section{References}

[1]. Hoffman JR, Mower WR, Wolfson AB, et al. Validity of a set of clinical criteria to rule out injury to the cervical spine in patients with blunt trauma. N Engl J Med. 2000

[2]. Barry TB, McNamara RM. Clinical decision rules and cervical spine injury in an elderly patient: a word of caution. J Emerg Med. 2005

[3]. Collins NC, McKenzie JV. The NEXUS criteria: do they withstand the test of time? Eur J Emerg Med. 2013

[4]. Morrison J, Jeanmonod R. Imaging in the NEXUS-negative patient: when we break the rule. Am J Emerg Med. 2014

[5]. Goode T, Young A, Wilson SP, et al. Evaluation of cervical spine fracture in the elderly: can we trust our physical examination? Am Surg. 2014

[6]. Touger M, Gennis P, Nathanson N, et al. Validity of a decision rule to reduce cervical spine radiography in elderly patients with blunt trauma. Ann Emerg Med. 2002

[7]. Matteucci MJ, Moszyk D, Migliore SA. Agreement between resident and faculty emergency physicians in the application of NEXUS criteria for suspected cervical spine injuries. J Emerg Med. 2015

[8]. Konstantinidis A, Plurad D, Barmparas G, et al. The presence of nonthoracic distracting injuries does not affect the initial clinical examination of the cervical spine in evaluable blunt trauma patients: a prospective observational study. J Trauma. 2011

[9]. Hustey FM, Meldon SW. The prevalence and documentation of impaired mental status in elderly emergency department patients. Ann Emerg Med. 2002

[10]. Hustey FM, Meldon SW, Smith D, et al. The effect of mental status screening on the care of elderly emergency department patients. Ann Emerg Med. 2003

[11]. Evans D, Vera L, Jeanmonod D, et al. Application of National Emergency X-Ray Utilizations Study low-risk c-spine criteria in high-risk geriatric falls. Am J Emerg Med. 2015

[12]. Michaleff ZA, Maher CB, Verhagen AP, et al. Accuracy of the Canadian C-spine Rule and NEXUS to screen for clinically important cervical spine injury in patients following blunt trauma: A systematic review. CMAJ

[13]. Centers for Disease Control and Prevention, National Center for Injury Prevention and Control. Web-based Injury Statistics Query and Reporting System (WISQARS)

[14]. Tinetti ME, Williams CS. Falls, injuries due to falls, and the risk of admission to a nursing home. N Engl J Med. 1997 